Research Article

\title{
Profiling Laboratory Biomarkers Associated with COVID-19 Disease Progression: A Single-Center Experience
}

\author{
Maria Khan (D), Noman Shah, Hina Mushtaq $(\mathbb{D}$, and Valeed Jehanzeb \\ Pathology Department, Rehman Medical Institute, Peshawar, Pakistan \\ Correspondence should be addressed to Maria Khan; kmaria22@hotmail.com
}

Received 7 November 2020; Revised 19 January 2021; Accepted 31 January 2021; Published 11 February 2021

Academic Editor: Faham Khamesipour

Copyright (C) 2021 Maria Khan et al. This is an open access article distributed under the Creative Commons Attribution License, which permits unrestricted use, distribution, and reproduction in any medium, provided the original work is properly cited.

\begin{abstract}
Background. There is clinical importance to investigate the disease progression through potential biomarkers of SARS-CoV-2 infection. In the present study, we aim to evaluate the significance of inflammatory markers in different categories of COVID-19 in admitted patients. Methods. In a single-center, observational study of 50 in-hospital patients who were diagnosed with COVID-19 and admitted to the intensive care unit of a tertiary care hospital in Peshawar, infection biomarkers, including hs-CRP, serum ferritin, serum creatinine, ALT, ALP, cardiac troponin-I, and IL-6 were analyzed. Results. The median age was 61.3 years. 70\% (35) were male while $30 \%$ (15) were female. We noted significantly increased hs-CRP $(9.32 \mathrm{mg} / \mathrm{dL} \pm 10.03)$ and ferritin levels $(982.3 \mathrm{ng} /$ $\mathrm{ml} \pm 601.9)$. A noteworthy increase was observed in neutrophil count $\left(11.05 \times 10^{9} / \mathrm{L}\right)$ and a decrease was observed in lymphocyte count $\left(0.27 \times 10^{9 /} \mathrm{L}\right)(P<0.05)$, and the platelet count was borderline decreased $\left(244.1 \times 10^{9} / \mathrm{L}\right)$. IL-6 levels were markedly increased in all admitted patients $(100.2 \mathrm{pg} / \mathrm{ml} \pm 122.2 \mathrm{pg} / \mathrm{ml})$. Conclusion. The serum levels of CRP, troponin-I, ALP, ALT, serum creatinine, and ferritin are markedly increased in COVID-19 patients. Increased CRP and ferritin levels were also associated with secondary bacterial infection and poor clinical outcomes.
\end{abstract}

\section{Introduction}

SARS-CoV-2 belongs to the Coronaviridae family of enveloped, positive-sense single-stranded RNA group of viruses, mainly causing respiratory, gastrointestinal, hepatic, and neurological manifestations [1]. SARS-CoV-2 belongs to group $2 \mathrm{~B}$ with around $70 \%$ resemblance to SARS-CoV-1 in nucleic acid sequence [2]. In the beginning, the laboratory findings depicted the cytopathic effect of SARS-CoV-2, leading to lung damage, apparent from pathological examination [3]. With disease progression, the lymphocyte count in the blood decreases drastically, along with an increase in the neutrophil count and a decrease in the platelet number, with prolonged activated thromboplastin time (APTT) and raised C-reactive protein, cardiac enzymes, and liver function tests. Inflammatory cytokine secretion (IL1RA, IL-1B, IL-6, IL-7, and IL-8) is associated with cytokine storm and contributes to the pathogenesis of severe cases of COVID-19 [4]. Usually, IL-6 is secreted from monocytes and interstitial fibroblasts or alveolar macrophages of lung tissues [5, 6]. Disease severity, e.g., disseminated intravascular coagulation (DIC), acute respiratory distress syndrome (ARDS) in lungs, cardiovascular injuries (ischemia, pulmonary thromboembolism, and deep venous thrombosis), and cerebral infarctions (embolism) due to SARS-CoV-2 virus, cannot be anticipated with certainty using laboratory biomarkers for D-dimer, prothrombin time (PT), and activated partial thromboplastin time (aPTT) [7]. Largely, clinical and laboratory parameters contributing to complications were elderly, dyspnea, decreasing oxygen saturation, aspartate aminotransferase (AST), elevated neutrophil count, gamma-glutamyltransferase levels, and lactate dehydrogenase, raised CRP, high serum ferritin level, and elevated interleukin-6 [8]. Specific blood parameters, such as lymphopenia, and certain chemical features, in particular, troponin-I, serum creatinine, alanine aminotransferase, and alkaline phosphatase were delineated to be linked with the severity of COVID-19 [9]. Notably, increasing serum CRP levels corresponded to disease progression, serving as an early predictor for COVID-19 complication, prior to 
indications of critical findings with CT scan [10]. Serum ferritin rises proportionately in reaction to the inflammatory process, a well-known biomarker in a variety of diseases; additionally, the infamous biomarker of neutrophil-tolymphocyte ratio (NLR) serves as a prognostic indicator for fatality in patients with different inflammatory manifestations [11]. Some studies have emphasized the role of highsensitivity cardiac troponin-I (hs-TnI) being a contributing factor to poor prognosis [12]. With a crude mortality rate of about $2.3 \%$ [13], therefore, the prompt spread of disease requires instant labeling of cases into risk group categories following laboratory-confirmed diagnosis, to make sure optimum resource provision and implement swift management protocols [14]. The biomarkers we review in this study are serum ferritin, IL-6, hs-CRP, white cell count (WCC), platelet count, aPTT, alkaline phosphatase (ALP), alanine aminotransferase (ALT), cardiac troponin-I, and renal markers. This study aimed at investigating the correlation of different laboratory inflammatory biomarkers among patients with COVID-19.

\section{Methodology}

The study was approved by the Institutional Ethical Board of Rehman Medical Institute, Peshawar. Total patients admitted to Rehman Medical Institute (Peshawar, Pakistan) between 17 June 2020 and 2 July 2020 with laboratory-confirmed SARSCoV-2 infection were $n=87$, among which 50 were included where the other 37 patients had either mild symptoms or asymptomatic admitted for some other noninfectious ailments. Demographic and epidemiological statistics, such as age, sex, and disease history, were gathered upon admittance. For laboratory confirmation, real-time reverse transcriptasepolymerase chain reaction (RT-PCR) was used as gold standard, according to the recommended protocol of the hospital. All baseline serum samples were collected immediately after admission for total blood count, ALT, ALP, CRP, aPTT, IL-6, and ferritin. For quality control, serum samples from 25 healthy volunteers were collected.

Patients were categorized into moderate, severe, and critical COVID-19 groups according to China's Novel Coronavirus Pneumonia Diagnosis and Treatment Guideline (5th edition) $[15,16]$.

2.1. Moderate Group. Moderate group included patients with fever, respiratory manifestations, and imaging findings of pneumonia.

2.2. Severe Group. Severe group included patients with the following additional findings: respiratory distress, $\mathrm{RR} \geq 30$ times/minute; oxygen saturation $\left(\mathrm{SpO}_{2}\right) \leq 93 \%$; oxygen partial pressure $\left(\mathrm{PaO}_{2}\right)$ /oxygen concentration $\left(\mathrm{FiO}_{2}\right)$ in arterial blood $\leq 300 \mathrm{mmHg}$; and $>50 \%$ lung imaging deteriorating within $24-48 \mathrm{~h}$.

2.3. Critical Group. Critical group included patients having any of the following findings: respiratory failure and mechanical ventilation essential; shock; multiorgan failure; and need for intensive care unit.

2.4. Laboratory Investigation. Roughly $2 \sim 4 \mathrm{ml}$ of peripheral blood was collected from subjects of the categorized groups, and serum was separated by $2000 \mathrm{rpm} / 20 \mathrm{~min}$ centrifugation. Serum cytokines were analyzed using the Elecsys IL-6 immunoassay and electrochemiluminescence immunoassay (ECLIA) performed on a COBAS e411 analyzer (Roche Ltd.). It is an in vitro diagnostic test for the quantitative determination of IL- 6 in human blood. $25 \mu$ l of serum was incubated with biotinylated monoclonal IL- 6 antibody, labeled with a ruthenium complex and streptavidin-coated microparticles, forming a sandwich complex with the antigens present in the sample, and the resulting chemiluminescence is then measured by using photomultiplier. Data were statistically analyzed by SPSS for Windows 25.0 (SPSS Inc., Chicago, IL). A value of $p<0.05$ was considered statistically significant.

\section{Results}

Among the total of 50 admitted patients, 30\% were classified as Critical group $(n=15), 48 \%$ were classified as Severe group $(n=24)$, and $22 \%(n=11)$ were classified as Moderate group. The mean age of patients was 61.3 years $(\mathrm{SD}=14.4)$. $70 \%$ (35) were male, while $30 \%$ (15) were female. Table 1 shows the mean value of biomarkers in total patients.

Duration for hospital admission to intubation period variegated from less than two hours to 9 days (median 4 days). Elevated interleukin-6 (IL-6), troponin-I, and ferritin levels were influentially accompanied by mechanical ventilation need. In total, 38/50 (68\%) patients deteriorated during hospitalization and required mechanical ventilation. The mortality rate was $92 \%$ among patients on mechanical ventilation. Table 2 shows the individual biomarker profile of each patient.

Among the hematologic parameters, total hemoglobin levels were low in $16 \%(n=8)$ of patients, total platelet count was low in $18 \%(n=9)$ of patients, total leukocyte count was high in $44 \%$ of patients, neutrophil count was high in $94 \%$ $(n=47)$ of patients, lymphocyte count was low in almost $50 \%(n=25)$ of patients, whereas neutrophil-to-lymphocyte ratio was increased in $92 \%(n=46)$ of patients admitted.

Cardiac markers, hs-troponin-I levels, were high in $72.5 \%(n=36)$, serum ferritin values were high in $96 \%$ $(n=48)$, serum alkaline phosphatase was high in $36 \%$ $(n=18)$, serum creatinine was high in $60 \%(n=30)$, CRP was high in $82 \%(n=41)$, and serum ALT was raised in $36 \%(18)$ of patients.

Similarly, serum ferritin levels directly correlated with increasing levels of serum ALP $(r=0.548, P<0.0001)$, serum troponin $(r=0.37, P<0.05)$, CRP $(r=0.306, P<0.05)$, and neutrophil count $(r=0.79, P<0.0001)$. Serum alkaline phosphatase positively correlated with levels of CRP values $(r=0.439, P<0.05)$ and neutrophil count $(r=0.47, P<0.05)$. There was a positive correlation of aPTT with neutrophil-tolymphocyte ratio $(r=0.45, P<0.005)$, TLC $(r=0.363$, 
TABLE 1: Mean value of inflammatory biomarkers.

\begin{tabular}{lcc}
\hline Variable & Mean & Standard deviation \\
\hline Serum interleukin-6 & $100.20 \mathrm{pg} / \mathrm{mL}$ & $122.2 \mathrm{pg} / \mathrm{mL}$ \\
Serum ferritin & $982.3 \mathrm{ng} / \mathrm{ml}$ & $601.9 \mathrm{ng} / \mathrm{ml}$ \\
Serum alkaline phosphatase (ALP) & $109.2 \mathrm{U} / \mathrm{L}$ & $41.1 \mathrm{U} / \mathrm{L}$ \\
Ultrasensitivity cardiac troponin-I & $2972.3 \mathrm{ng} / \mathrm{dL}$ & $7615.8 \mathrm{ng} / \mathrm{dL}$ \\
Serum creatinine & $2.09 \mathrm{mg} / \mathrm{dL}$ & $1.80 \mathrm{mg} / \mathrm{dL}$ \\
Serum C-reactive protein (CRP) & $9.32 \mathrm{mg} / \mathrm{dL}$ & $10.03 \mathrm{mg} / \mathrm{dL}$ \\
Serum alanine aminotransferase (ALT) & $48.94 \mathrm{U} / \mathrm{L}$ & $38.49 \mathrm{U} / \mathrm{L}$ \\
Activated partial thromboplastin time (APTT) & $31.76 \mathrm{~seconds}$ & $21.52 \mathrm{~seconds}$ \\
Hemoglobin levels (Hb) & $12.54 \mathrm{~g} / \mathrm{dL}$ & $2.2 \mathrm{~g} / \mathrm{dL}$ \\
Total leukocyte count (TLC) & $12.97 \times 10^{6} / \mu L$ & $8.04 \times 10^{6} / \mu L$ \\
Platelet count & $244.1 \times 10^{9} / \mathrm{L}$ & $121.72 \times 10^{9} / \mathrm{L}$ \\
Neutrophil count & $11.0502 \times 10^{9} / \mathrm{L}$ & $2.76044 \times 10^{9} / \mathrm{L}$ \\
Lymphocyte count & $0.2754 \times 10^{9} / \mathrm{L}$ & $1.02173 \times 10^{9} / \mathrm{L}$ \\
Neutrophil-to-lymphocyte ratio (NLR) & 15.6 & 16.50 \\
\hline
\end{tabular}

$P<0.05)$, and hs-CRP $(r=0.339, P<0.05)$. In contrast, negative correlation of platelet count with IL-6, serum creatinine, ALP, hs-troponin-I, TLC, serum ALT, and serum CRP was noted. Lymphocyte count also negatively correlated with IL-6, serum ferritin, ALP, CRP, aPTT values, serum creatinine, and hemoglobin levels.

\section{Discussion}

The documentation of prognostic variables may contribute to the evidence-based management of patients with COVID19. In this study, we evaluated the demographic, diagnostic, and clinical characteristics of 50 patients with COVID-19 admitted to ICU and determined probable inflammatory markers affecting the clinical outcomes of patients. It should be noted that biomarkers are used in the diagnosis of various clinical conditions reflecting pathological progression. We established ALT elevation in 36\% of cases, CRP was raised in $82 \%$, alkaline phosphatase was raised in $36 \%$, and $96 \%$ of patients had markedly increased serum ferritin levels. Moreover, in severe and critical categories, IL-6 levels were markedly raised. Particularly, this study indicated inflammatory events due to cytokine storms, high CRP, and markedly raised serum ferritin levels among critically ill patients contributing to worsened disease state, clearly highlighting the management of lung damage as a crucial step. Apart from this, our findings suggest that the expression of IL- 6 and CRP accounts for timely diagnosis of patients having severe disease, keeping in view the substantial burden of healthcare in individual hospitals. Subsequently, hyperferritinemic cases were among the male and elderly age group, correlating towards more severe disease than those with normal serum ferritin levels. Besides, these particular patients had considerably raised serum creatinine, ALP, and ALT, decreased lymphocyte count, and increased levels of inflammatory biomarkers, such as serum CRP [17]. The association of hyperferritinemia and complications in patients with SARS-CoV-2 is indistinguishable, but the contributing factors such as IL-6 directly affecting ferritin synthesis can be a possibility [18, 19]. Similarly, ferritin functions as iron binding and storage and is indirectly associated with immunity and inflammatory responses of the body [20]. The link between CRP and COVID-19 has been emphasized by a study at a tertiary care hospital in Wuhan, where most of the patients in the severe category exhibited increased parameters in comparison with the nonsevere group (57.9-33.2 mg/L) [21]. Similarly, another study established disease progression to a severe state due to CRP levels of $>41.8 \mathrm{mg} / \mathrm{L}$ [22]. In critically ill patients, persistently raised IL- 6 levels released by activated macrophages correlate directly to high viral RNA load which in turn leads to ARDS deterioration [23-25]. Comparatively, platelet count was significantly decreased in critically ill patients and nonsurvivor than survivors [26]. Yang et al. in one of his studies reported lymphopenia among $80 \%$ of critically ill patients [6], while Chen et al. stated lymphopenia in $25 \%$ of patients having mild infection [24]. However, in the present study, $50 \%$ of patients suffered from lymphopenia. Interestingly, the levels of other white cell counts, monocytes, eosinophils, and basophils, also decreased, ensuing greater neutrophil-to-lymphocyte ratio that portrays poor prognosis [27]. In our study, the mean NLR was 15.6 and was significantly raised in $92 \%$ of patients. A meta-analytic study revealed significantly lower platelet counts in 1799 patients with severe COVID-19 infections [28].

In addition, cardiac enzymes and muscular biomarkers were also raised in severe and critical COVID-19 patients. Another prospective study of 179 patients with COVID-19 reported cardiac troponin-I $\geq 0.05 \mathrm{ng} / \mathrm{mL}$, amongst high risk factors and predictors of mortality [29]. Similarly, we found $72.5 \%$ of patients having elevated hs-troponin-I, with a mean value of $2972.3 \mathrm{ng} / \mathrm{dl}$. In the same way, 416 hospitalized patients of COVID-19 reported high-sensitivity troponin-I elevation among 1 in 5 patients on admission [30]. Timely recognition of cardiac injury by deranged hs-TnI levels supports incorrect triage measures and directs towards the usage of vasopressors and inotropes. Significantly raised levels of serum urea and creatinine also contribute to disease severity [31]. In a wide retrospective multicenter study involving 5771 patients with COVID-19, Lei et al. reported a strong association of serum ALT levels with mortality risk 


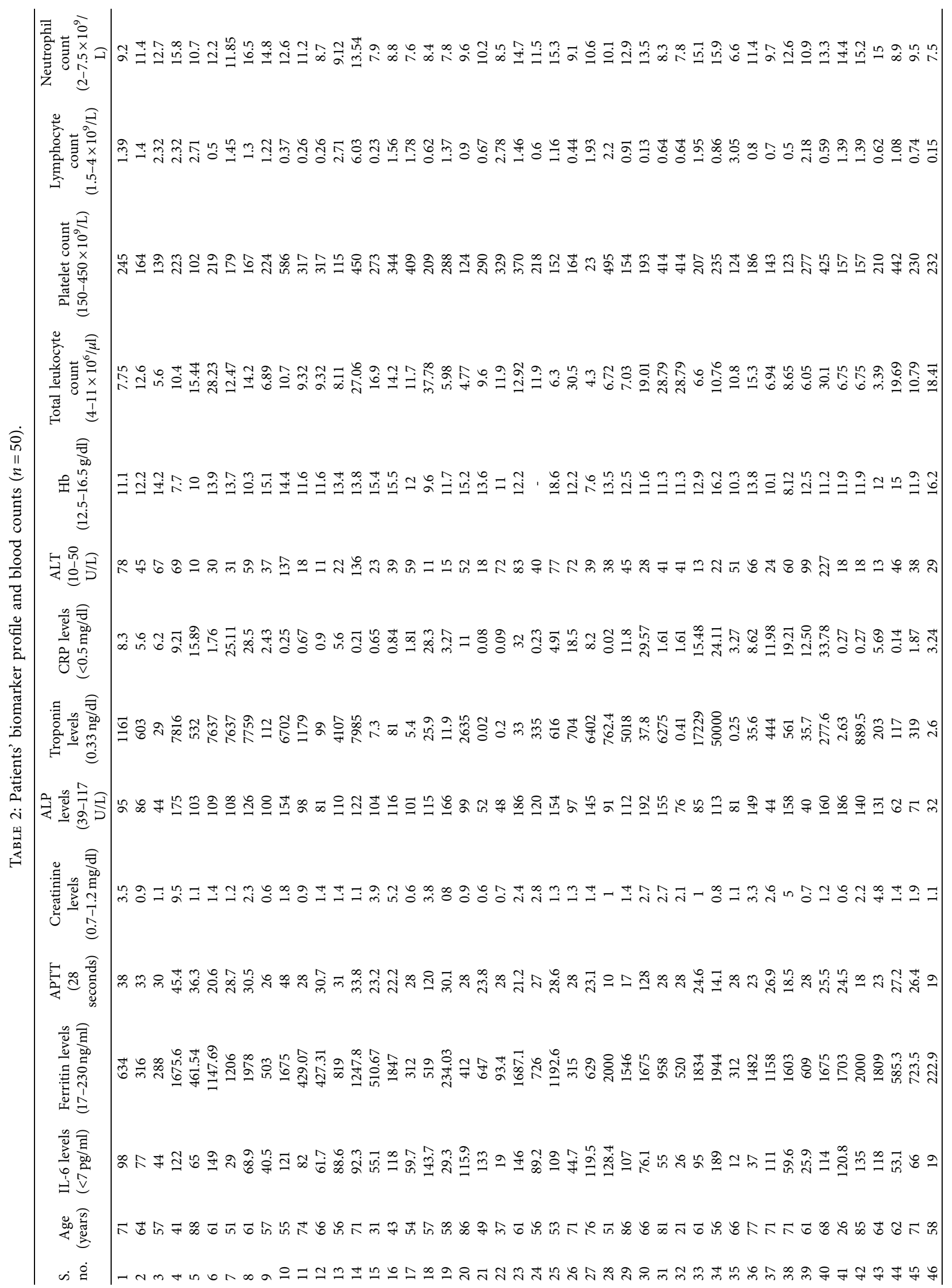




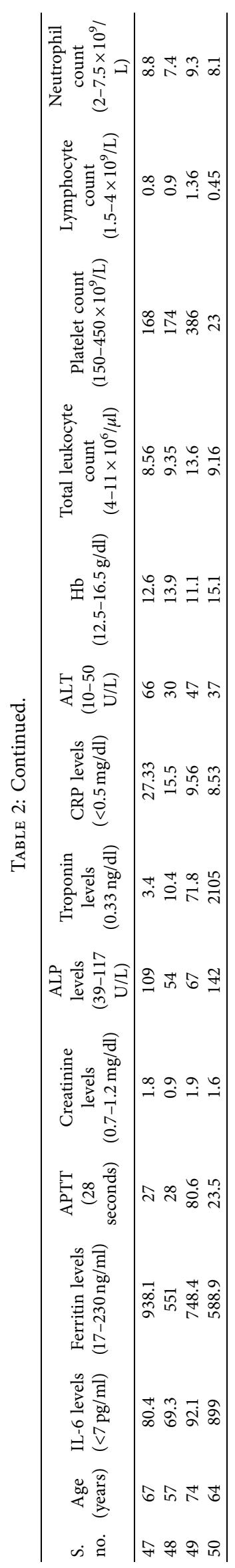


compared to other liver injury parameters [32]. In the present study, $36 \%$ of patients had elevated serum ALT levels, with a mean of $48.94 \mathrm{U} / \mathrm{L}$.

\section{Conclusion}

From the time when the pandemic crisis originated, it is of utmost importance to analyze the clinical implication of hematologic, biochemical, inflammatory, and immunologic biomarkers in patients with or without severe/fatal forms of COVID-19. Inference gathered till date suggests the strong clinical correlation among varied levels of inflammatory markers that confer to the severity of COVID-19, alternatively being used as an adjunct in guiding towards treatment options and criteria for admission, thereby improving prognosis and minimizing mortality rate. Our findings indicated that C-reactive protein level, serum ferritin, and troponin-I were autonomous predictors of severity in COVID-19 cases. Independently, lymphocyte count has been recommended as a probable biomarker of the disease, showing consistently low levels reported in critically ill patients. Elevated serum CRP as well as ferritin levels might have a clinical correlation with secondary bacterial infection, contributing to poor prognosis.

\section{Abbreviations}

aPTT: $\quad$ Activated partial thromboplastin time

ARDS: $\quad$ Acute respiratory distress syndrome

ALT: Alanine aminotransferase

ALP: Alkaline phosphatase

CDC: $\quad$ Centers for Disease Control and Prevention

COVID-19: 2019 novel coronavirus disease

DIC: Disseminated intravascular coagulation

hs-CRP: High-sensitivity C-reactive protein

ICU: Intensive care unit

IL-6: Interleukin-6

NLR: $\quad$ Neutrophil-to-lymphocyte ratio

$\mathrm{PO}_{2}$ : $\quad$ Partial pressure of oxygen

PT: $\quad$ Prothrombin time

RNA: $\quad$ Ribonucleic acid

$\mathrm{SpO}_{2}$ : Oxygen saturation

SARS-CoV- Severe acute respiratory syndrome

2: $\quad$ coronavirus 2

TLC: $\quad$ Total leukocyte count.

\section{Data Availability}

The data used to support the findings of this study are included within the article.

\section{Ethical Approval}

The study was approved by the Ethics Committee of Rehman Medical Institute, Peshawar.

\section{Consent}

Written informed consent was obtained from all participants.

\section{Conflicts of Interest}

The authors declare that they have no competing interests.

\section{Authors' Contributions}

MK collected the clinical and laboratory data and revised the final manuscript. NS processed statistical analysis. HM drafted the manuscript. VJ performed data analysis.

\section{References}

[1] S. R. Weiss and J. L. Leibowitz, "Coronavirus pathogenesis," Advances in Virus Research, Academic Press, vol. 81, pp. 85-164, 2011.

[2] D. S. Hui, E. I Azhar, T. A. Madani et al., "The continuing 2019-nCoV epidemic threat of novel coronaviruses to global health - the latest 2019 novel coronavirus outbreak in Wuhan, China," International Journal of Infectious Diseases, vol. 91, pp. 264-266, 2020.

[3] Z. Xu, L. Shi, Y. Wang et al., "Pathological findings of COVID19 associated with acute respiratory distress syndrome," The Lancet Respiratory Medicine, vol. 8, no. 4, pp. 420-422, 2020.

[4] J. She, J. Jiang, L. Ye, L. Hu, C. Bai, and Y. Song, "Novel coronavirus of pneumonia in Wuhan, China: emerging attack and management strategies," Clinical and Translational Medicine, vol. 9, no. 1, pp. 1-7, 2020.

[5] R. M. Kotloff, J. Little, and J. A. Elias, "Human alveolar macrophage and blood monocyte interleukin-6 production," American Journal of Respiratory Cell and Molecular Biology, vol. 3, no. 5, pp. 497-505, 1990.

[6] X. Yang, Y. Yu, J Xu et al., "Clinical course and outcomes of critically ill patients with SARS-CoV-2 pneumonia in Wuhan, China: a single-centered, retrospective, observational study," The Lancet Respiratory Medicine, vol. 8, no. 5, 2020.

[7] C. Huang, Y. Wang, X. Li et al., "Clinical features of patients infected with 2019 novel coronavirus in Wuhan, China," The Lancet, vol. 395, no. 10223, pp. 497-506, 2020.

[8] Y. Sun, Y. Dong, L. Wang et al., "Characteristics and prognostic factors of disease severity in patients with COVID-19: the Beijing experience," Journal of Autoimmunity, vol. 112, Article ID 102473, 2020.

[9] G. Zhang, J. Zhang, B. Wang, X. Zhu, Q. Wang, and S. Qiu, "Analysis of clinical characteristics and laboratory findings of 95 cases of 2019 novel coronavirus pneumonia in Wuhan, China: a retrospective analysis," Respiratory Research, vol. 21, no. 1, p. $1,2020$.

[10] C. Tan, Y. Huang, F Shi et al., "C-reactive protein correlates with computed tomographic findings and predicts severe COVID-19 early," Journal of Medical Virology, vol. 92, no. 7, pp. 856-862, 2020.

[11] T. Bhat, S. Teli, J. Rijal et al., "Neutrophil to lymphocyte ratio and cardiovascular diseases: a review," Expert Review of Cardiovascular Therapy, vol. 11, no. 1, pp. 55-59, 2013.

[12] S. Zaim, J. H. Chong, V. Sankaranarayanan, and A. Harky, "COVID-19 and multiorgan response," Current Problems in Cardiology, vol. 45, no. 8, Article ID 100618, 2020.

[13] C. P. Novel, "The epidemiological characteristics of an outbreak of 2019 novel coronavirus diseases (COVID-19) in China," Zhonghua Liu Xing Bing Xue Za Zhi, vol. 41, no. 2, pp. 145-151, 2020.

[14] G. Ponti, M. Maccaferri, A. Percesepe, A. Tomasi, and T. Ozben, "Liquid biopsy with cell free DNA: new horizons for 
prostate cancer," Critical Reviews in Clinical Laboratory Sciences, vol. 57, no. 8, pp. 1-17, 2020.

[15] L. Lin and T. S. Li, "Interpretation of " guidelines for the diagnosis and treatment of novel coronavirus (2019-ncov) infection by the national health commission (trial version 5)," Zhonghua Yi Xue Za Zhi, vol. 100, p. E001, 2020.

[16] Y. H. Jin, L. Cai, Z. S Cheng et al., "A rapid advice guideline for the diagnosis and treatment of 2019 novel coronavirus (2019nCoV) infected pneumonia (standard version)," Military Medical Research, vol. 7, no. 1, p. 4, 2020.

[17] Z. Lin, F. Long, Y. Yang, X. Chen, L. Xu, and M. Yang, "Serum ferritin as an independent risk factor for severity in COVID19 patients," Journal of Infection, vol. 81, no. 4, pp. 647-679, 2020.

[18] M. Kobune, Y. Kohgo, J. Kato, E. Miyazaki, and Y. Niitsu, "Interleukin-6 enhances hepatic transferrin uptake and ferritin expression in rats," Hepatology, vol. 19, no. 6, pp. 1468-1475, 1994.

[19] D. B. Kell, "Iron behaving badly: inappropriate iron chelation as a major contributor to the aetiology of vascular and other progressive inflammatory and degenerative diseases," $B M C$ Medical Genomics, vol. 2, no. 1, pp. 1-79, 2009.

[20] A. Lalueza, B. Ayuso, E Arrieta et al., "Elevation of serum ferritin levels for predicting a poor outcome in hospitalized patients with influenza infection," Clinical Microbiology and Infection, vol. 26, no. 11, pp. 1557.e9-1557.e15, 2020.

[21] C. Qin, M. P. Ziwei, S. Y. Tao, P. C. Ke, and M. M. Shang, "Dysregulation of immune response in patients with COVID19 in wuhan, China; clinical infectious diseases," Clinical Infectious Diseases, vol. 71, no. 15, pp. 762-768.

[22] F. Liu, L. Li, M. Xu et al., "Prognostic value of interleukin-6, C-reactive protein, and procalcitonin in patients with COVID-19," Journal of Clinical Virology, vol. 127, Article ID 104370, 2020

[23] X. Chen, B. Zhao, Y Qu et al., "Detectable serum SARS-CoV-2 viral load (RNAaemia) is closely correlated with drastically elevated interleukin 6 (IL-6) level in critically ill COVID-19 patients," Clinical Infectious Diseases, vol. 449, 2020.

[24] N. Chen, M. Zhou, X. Dong et al., "Epidemiological and clinical characteristics of 99 cases of 2019 novel coronavirus pneumonia in Wuhan, China: a descriptive study," The Lancet, vol. 395, no. 10223, pp. 507-513, 2020.

[25] E. A. Coomes and H. Haghbayan, "Interleukin-6 in COVID19: a systematic review and meta-analysis," MedRxiv, vol. 30, no. 6, pp. 1-9, 2020.

[26] N. Tang, H. Bai, X. Chen, J. Gong, D. Li, and Z. Sun, "Anticoagulant treatment is associated with decreased mortality in severe coronavirus disease 2019 patients with coagulopathy," Journal of Thrombosis and Haemostasis, vol. 18, no. 5, pp. 1094-1099, 2020.

[27] L. Tan, Q. Wang, D Zhang et al., "Lymphopenia predicts disease severity of COVID-19: a descriptive and predictive study," Signal Transduction and Targeted Therapy, vol. 5, no. 1, 33 pages, 2020.

[28] G. Lippi, M. Plebani, and B. M. Henry, "Thrombocytopenia is associated with severe coronavirus disease 2019 (COVID-19) infections: a meta-analysis," Clinica Chimica Acta, vol. 506, pp. 145-148, 2020.

[29] R. H. Du, L. R. Liang, C. Q Yang et al., "Predictors of mortality for patients with COVID-19 pneumonia caused by SARSCoV-2: a prospective cohort study," European Respiratory Journal, vol. 55, no. 5, 2020.

[30] S. Shi, M. Qin, B Shen et al., "Association of cardiac injury with mortality in hospitalized patients with COVID-19 in
Wuhan, China," JAMA Cardiology, vol. 5, no. 7, pp. 802-810, 2020.

[31] J. Xiang, J. Wen, X Yuan et al., "Potential biochemical markers to identify severe cases among COVID-19 patients," MedRxiv, 2020.

[32] F. Lei, Y. M. Liu, F Zhou et al., "Longitudinal association between markers of liver injury and mortality in COVID-19 in China," Hepatology, vol. 72, no. 2, pp. 389-398, 2020. 